\title{
THE BELLATERRA CONNECTION
}

\author{
JAAK PEETRE
}

\begin{abstract}
A new object is introduced - the "Fischer bundle". It is, formally speaking, an Hermitean bundle of infinite rank over a bounded symmetric domain whose fibers are Hilbert spaces whose elements can be realized as entire analytic functions square integrable with respect to a Gaussian measure ("Fischer spaces"). The definition was inspired by our previous work on the "Fock bundle". An even more general framework is indicated, which allows one to look upon the two concepts from a unified point of view.
\end{abstract}

0. Introduction. The underlying idea of this research is the following: Let $\mathcal{D}$ be a circular bounded symmetric domain contained in a vector space $V$. The usual Fischer space corresponding to a symmetric domain (cf. e. g. [1]) depends on the Harish-Chandra realization, that is, the choice of a base point; in the case at hand it is the origin. So, if we make the base point vary, we get a whole family of Fischer spaces, in other words, an Hermitean vector bundle of infinite rank over the given symmetric domain, the Fischer bundle. In the same way, starting with the Bergman space, one is lead to the Bergman bundle. This situation is analogous to and inspired by our previous construction of the Fock bundle (see $[\mathbf{4}],[\mathbf{5}],[\mathbf{6}],[\mathbf{7}]$ ). However, as we have so far not found any very convicing applications, we have been forced to draw the conclusion that the Fischer and the Bergman are poor relatives of the Fock bundle, rather poor indeed... One application is, though, a rather unexpected one, to the holonomy group of $\mathcal{D}$ (see the remark at the end of Section 4). In Section 5 we propose a general framework which allows one to look at the Fischer bundle and the Fock bundle from a unified point of view.

Acknowledgement. I would like to thank Profs. J. Bruna and $M$. Castellet, and all my other friends and colleagues at the Centre de Recerca Matemàtica and the Departament de Matemàtiques of the Universitat Autònoma de Barcelona for hospitality and for providing excellent working conditions during the months of May and June, 1992. 
1. The case of the disk. In this Section we take $\mathcal{D}=\{|z|<1\}=$ unit disk, $V=\mathbb{C}=$ complex plane. We define the Fischer bundle $F$ over $\mathcal{D}$ to be the Hermitean bundle of infinite rank (Hilbert bundle) such that the fiber $F_{z}$ at a point $z \in \mathcal{D}$ is the Hilbert space of entire analytic functions $f=f(v)$ (with $v \in \mathbb{C}(=V)$ ) such that the norm

$$
\|f\|_{z}^{2}=\int_{\mathbb{C}}|f(v)|^{2} \frac{e^{-\frac{|v|^{2}}{\left(1-|z|^{2}\right)^{2}}}}{\left(1-|z|^{2}\right)^{2}} d m(v)<\infty,
$$

where $d m$ is Lebesgue measure in $\mathbb{C}$ normalized in such a way that the area of $\mathcal{D}$ is one. We denote the corresponding inner product by $(f, g)_{z}$.

Motivation: If we make the usual identification $T_{z} \mathcal{D} \approx \mathbb{C}$ (for $z \in$ $D$ ), the square of the length of a tangent vector $v$ at a point $z$ in the hyperbolic (or non-Euclidean) metric is

$$
\frac{|v|^{2}}{\left(1-|z|^{2}\right)^{2}}
$$

Similarly,

$$
\frac{d m(v)}{\left(1-|z|^{2}\right)^{2}}
$$

is the hyperbolic (or Poincaré) measure.

Let us identify the Hermitean connection in $F$. (Recall that an Hermitean connection on an Hermitean vector bundle is an operation $\nabla$ on smooth sections of the bundle which satisfies a number of properties, the two most important of which being

$$
\begin{aligned}
d\|f\|^{2} & =(\nabla f, f)+(f, \nabla f), \\
\nabla^{\prime \prime} f & =d^{\prime \prime} f ;
\end{aligned}
$$

we use a double prime to denote the anti-holomorphic parts of $\nabla$ and exterior derivative $d$ respectively).

Denoting the weight function occuring in (1) by $W$,

$$
W=W(z, v)=\frac{e^{-\frac{|v|^{2}}{\left(1 n|z|^{2}\right)^{2}}}}{\left(1-|z|^{2}\right)^{2}}
$$

and differentiating, yields

$$
\frac{\partial}{\partial z}\|f\|_{z}^{2}=\int_{\mathbb{C}}\left(\frac{\partial f}{\partial z} \bar{f} W+f \frac{\overline{\partial f}}{\partial \bar{z}} W+f \bar{f} \frac{\partial W}{\partial z}\right) .
$$


Lemma. We have

$$
\frac{\partial W}{\partial z}=\frac{2 \bar{z}}{1-|z|^{2}} \frac{\partial(v W)}{\partial v}
$$

Proof: One readily finds

$$
\begin{aligned}
& \frac{\partial W}{\partial z}=W\left[-\frac{2 \bar{z}|v|^{2}}{\left(1-|z|^{2}\right)^{3}}+\frac{2 \bar{z}}{1-|z|^{2}}\right] \\
& \frac{\partial W}{\partial v}=W\left[\frac{\bar{v}}{\left(1-|z|^{2}\right)^{2}}\right] .
\end{aligned}
$$

Thus by elimination of $|v|^{2}$ between the above equation we obtain

$$
\frac{\partial W}{\partial z}=\frac{2 \bar{z}}{1-|z|^{2}}\left(v \frac{\partial W}{\partial v}+W\right)
$$

As

$$
v \frac{\partial W}{\partial v}+W=\frac{\partial(v W)}{\partial v},
$$

this completes the proof.

Integrating by parts in (2) and using the Lemma gives

$$
\frac{\partial\|f\|_{z}^{2}}{\partial z}=\left(\frac{\partial f}{\partial z}-\frac{2 \bar{z}}{1-|z|^{2}} v \frac{\partial f}{\partial v}, f\right)_{z}+\left(f, \frac{\partial f}{\partial \bar{z}}\right)_{z},
$$

From this we may infer the expression for the connection.

Proposition. We have

$$
\nabla f=d f-\frac{2 \bar{z} d z}{1-|z|^{2}} v \frac{\partial f}{\partial v}
$$

In other words, the rôle of the connection form is played by the (operator valued) differential form

$$
\omega=-\frac{2 \bar{z} d z}{1-|z|^{2}} v \frac{\partial}{\partial v}
$$

Similarly, using (3) we can easily determine the corresponding curvature form. 
Corollary. The curvature form is given by

$$
\Omega=\frac{2 d z d \bar{z}}{\left(1-|z|^{2}\right)^{2}} v \frac{\partial}{\partial v}
$$

Thus we may draw the conclusion that the connection is not flat.

Proof: The simplest way to derive this expression is to seek the integrability conditions for the equation $\nabla f=0$. Differentiating this equation we find with the aid of (3)

$$
0=-d\left(\frac{2 \bar{z} d z}{1-|z|^{2}}\right) v \frac{\partial f}{\partial v},
$$

which readily leads to $(4)$.

2. Parallel transport (still for the disc). Let $z=z_{t}=z(t)$ be an arc in $D$ issuing from the origin $\left(z_{0}=0\right)$ and let $f \in F_{0}$ (the fiber of $F$ at this point). We seek its horizontal lift $f_{t}$ to $F$ through the element $f\left(f_{t} \in F_{t}, f_{0}=f\right)$. We make the "Ansatz"

$$
f_{t}(v)=f(\phi(t) v)
$$

where $\phi$ is a scalar function. Differentiation of (1) yields

$$
\begin{aligned}
\frac{d}{d t} f_{t}(v) & =f^{\prime} \phi^{\prime} v \\
v \frac{\partial}{\partial v} f_{t}(v) & =f^{\prime} \phi v .
\end{aligned}
$$

Moreover, obviously $d z(t)=z^{\prime}(t) d t$. Thus we find with the help of (2) in Section 1

$$
0=\nabla f_{t}=f^{\prime}\left(\phi^{\prime}-\frac{2 \overline{z(t)} z^{\prime}(t)}{1-|z(t)|^{2}} \phi\right) v d t .
$$

Hence the function $\phi$ can be determined by solving the ordinary differential equation

$$
\phi^{\prime}=\frac{\overline{2 z(t)} z^{\prime}(t)}{1-|z(t)|^{2}} \phi
$$

with initial condition $\phi(0)=1$.

Let us look at two examples. 
Example 1. Let us consider the segment $(0,1)$ (a geodesic ray in the hyperbolic metric) parametrized by the identity map, $z_{t}=t(t \in(0,1))$. Thus in this case

$$
\phi^{\prime}=\frac{2 t}{1-t^{2}} \phi
$$

yielding

$$
\phi(t)=\frac{1}{1-t^{2}}
$$

so that

$$
f_{t}(v)=f\left(\frac{v}{1-t^{2}}\right)
$$

Example 2. Consider a concentric circle of radius $r$ with the parametrization $z=r e^{i t}$. Then

$$
\phi^{\prime}=\frac{2 r i}{1-r^{2}} \phi
$$

yielding

$$
\phi(t)=e^{i \frac{2 r t}{1-r^{2}}}
$$

and so

$$
f_{t}(v)=f\left(e^{i \frac{2 r t}{1-r^{2}}} v\right)
$$

in this case.

Alternatively, we could also have solved the same problem by the method of separation of variables. Indeed, setting $f_{t}(v)=a_{n}(t) v^{n}$ leads to the ordinary differential equation

$$
a_{n}^{\prime}=\frac{2 n \overline{z(t)} z^{\prime}(t)}{1-|z(t)|^{2}} a_{n}
$$

with the solution

$$
a_{n}(t)=\exp \left(2 n \int_{0}^{t} \frac{\overline{z(\tau)} z^{\prime}(\tau)}{1-|z(\tau)|^{2}} d \tau\right) a_{n}(0) .
$$

Remark. The above indicates also that the Fischer bundle $F$ comes as the direct sum of certain lines bundles $F(n)(n=0,1, \ldots)$ over $\mathcal{D}$,

$$
F=\sum_{n=0}^{\infty} \oplus F(n)
$$


Indeed, let the function $f=f(z, v)$ represent a section of $F$. We write its Taylor series in the variable $v$,

$$
f(z, v)=\sum_{n=0}^{\infty} a_{n}(z) v^{n} .
$$

Then the coefficients $a_{n}(z)$ correspond to differential forms $a_{n}(z)(d z)^{n}$, while $v$ corresponds to a tangent vector at the point $z$. For the Moebius group $\mathbf{G}_{0}=\mathrm{PU}(1,1)=\mathrm{SU}(1,1) / \pm 1$ acts on $F$ via

$$
\begin{aligned}
& f(z, v) \mapsto f\left(\frac{\alpha z+\beta}{\gamma z+\delta}, \frac{v}{(\gamma z+\delta)^{2}}\right) \equiv g(z, v) \\
& \quad\left(\text { with } \varphi=\left(\begin{array}{cc}
\alpha & \beta \\
\gamma & \delta
\end{array}\right) \in \mathrm{SU}(1,1)\right),
\end{aligned}
$$

where the right hand side has the expansion

$$
g(z, v)=\sum_{n=0}^{\infty} a_{n}\left(\frac{\alpha z+\beta}{\gamma z+\delta}\right) \frac{v^{n}}{(\gamma z+\delta)^{2 n}} ;
$$

that is, we have the rule

$$
a_{n}(z) \mapsto a_{n}\left(\frac{\alpha z+\beta}{\gamma z+\delta}\right) \frac{1}{(\gamma z+\delta)^{2 n}}
$$

or

$$
a_{n}(z)(d z)^{n} \mapsto a_{n}(\varphi(z))(d \varphi(z))^{2 n},
$$

with $\varphi(z)=\frac{\alpha z+\beta}{\gamma z+\delta}$.

It is casily seen directly that if $f$ represents a horizontal section $(\nabla f=$ 0 ) then so does $g(\nabla g=0)$. But this follows also from general theory, as the maps (3) are norm preserving (sce [4]-[7], where perhaps less trivial applications of the same general principle may be found).

Here are the details anyhow. Given $f$ let $g$ be as in (3). Then we find

$$
\begin{aligned}
& \frac{\partial g}{\partial z}=\frac{\partial f}{\partial z} \frac{1}{(\gamma z+\delta)^{2}}+\frac{\partial f}{\partial v} \frac{-2 \gamma v}{(\gamma z+\delta)^{3}}, \\
& \frac{\partial g}{\partial v}=\frac{\partial f}{\partial z} \frac{1}{(\gamma z+\delta)^{2}} .
\end{aligned}
$$

Hence

$$
\frac{\partial g}{\partial z}-\frac{2 \bar{z}}{1-|z|^{2}} v \frac{\partial g}{\partial v}=\left\{\frac{\partial f}{\partial z}-\frac{\partial f}{\partial v}\left[\frac{2 \gamma v}{\gamma z+\delta}+\frac{2 \bar{z}}{1-|z|^{2}} v\right]\right\} \frac{1}{(\gamma z+\delta)^{2}} .
$$

It is readily seen that the expression within brackets [ ] can be written as

$$
\frac{2 \overline{\varphi z}}{1-|\varphi z|^{2}} \frac{v}{(\gamma z+\delta)^{2}}
$$


3. Bergman bundle. If we instead of the Fischer metric start with the $\alpha$-Bergman metric, we get the $\alpha$-Bergman bundle $B^{\alpha}$. It has a theory entirely parallel to the one in Section 1-2. E. g. the fiber norm is given by

$$
\|f\|_{z}^{2}=\int_{|v|<1-|z|^{2}}|f(v)|^{2} \frac{\left(\left(1-\frac{|v|^{2}}{\left(1-|z|^{2}\right)^{2}}\right)^{2}\right)^{\alpha}}{\left(1-|z|^{2}\right)^{2}} d m(v) .
$$

Thus $f$ is not anymore an entire function. Another difference is that the connection form now corresponds to a differential operator of infinite order.

Remark. Note that in the flat limit (radius of curvature $\rightarrow \infty$ ) all these bundles give rise to a trivial connection $(\nabla f \equiv d f)$.

4. General symmetric domains. In this Section we let thus the base space be an arbitrary circular bounded symmetric domain which we again denote by the letter $\mathcal{D}$. The containing vector space (of finite dimension over $\mathbb{C}$ ) will be denoted by $V$. We consider only the Fischer bundle, leaving the $\alpha$-Bergman bundles alone.

We will use freely notation and results (with slight variations) in the excellent book [2]. In particular, let us remind that $Q(z): \bar{V} \mapsto V$ (where $z \in V$ ) is the quadratic map in the Jordan pair $(V, \bar{V})$ associated with the domain $\mathcal{D}(\bar{V}$ is the opposite vector space). By polarization one defines the maps $Q(z, w)$ and $D(z, \bar{\zeta})$, as well as the Jordan triple product $\{z, \bar{\zeta}, w\}: V \times \bar{V} \times V$ (which will not be used here),

$$
\{z, \bar{\zeta}, w\}=Q(z+w) \bar{\zeta}-Q(z) \bar{\zeta}-Q(w) \bar{\zeta}=Q(z, w) \bar{\zeta}=D(z, \bar{\zeta}) z .
$$

The Bergman operator is defined by the formula

$$
B(z, \bar{\zeta})=1-D(z, \bar{\zeta})+Q(z) Q(\bar{\zeta})
$$

The quasi-inverse of an element $z \in V$ with respect to an element $\bar{\zeta} \in \bar{V}$ will be written $z^{\bar{\zeta}}$,

$$
z^{\bar{\zeta}}=B^{-1}(z, \bar{\zeta})(z-Q(z) \bar{\zeta}) .
$$

The elements $f$ of the fiber $F_{z}$ at the point $z \in \mathcal{D}$ are now represented by entire functions $f(v)$ of the variable $v \in V$. The definition of the fiber norm should apparently read

$$
\|f\|_{z}^{2}=\int_{V}|f(v)|^{2} e^{-\left(B^{-1}(z, \bar{z}) v, \tilde{v}\right)} \operatorname{det}\left(B^{-1}(z, \bar{z})\right) d m(v) .
$$


Here $\left(\cdot,{ }^{\circ}\right)$ is the Hermitean inner product induced in $V$ from the Bergman metric in $\mathcal{D}$ in the usual identification $T_{0} \mathcal{D} \approx V$, while $d m$ is the corresponding Lebesgue measure, again normalized $(m(D)=1)$. The group $\mathbf{G}_{0}=$ (the identity component of the group Aut $\mathcal{D}$ of biholomorphic maps of $\mathcal{D}$ on to itself) acts on sections $f(z, v)$ of $F$ via

$$
f(z, v) \mapsto f\left(\varphi z,\left(\varphi^{\prime} z\right) v\right) \quad\left(\varphi \in \mathbf{G}_{0}\right) .
$$

It is clear that these maps are norm preserving. The Hermitean connection involved can be determined in an analogous way as in Section 1.

Indeed, corresponding to formula (1) there we have

$$
d_{z}^{\prime}\|f\|_{z}^{2}=\int_{Z}\left(d_{z}^{\prime} f \bar{f} W+f \overline{d_{z}^{\prime \prime}} W+d_{z}^{\prime} W\right) d m
$$

while the counterpart of the lemma reads.

Lemma. We have

$$
d_{z}^{\prime} W=d_{v}^{t} W\left(D\left(v, \overline{z^{\bar{z}}}\right) d z\right)+W\left(d z, \overline{z^{\bar{z}}}\right)
$$

(Here we use subscripts to indicate with respect to which variable we differentiate, while $z^{\bar{z}}$ denotes the quasi-inverse of $z$ with respect to $\bar{z}$, $W$ being the wcight factor in (1). We write $d=d^{\prime}+d^{\prime \prime}$; where $d^{\prime}$ and $d^{\prime \prime}$ are the holomorphic and the anti-holomorphic parts respectively of the exterior derivative $d$, that is, $d^{\prime}=\partial, d^{\prime \prime}=\bar{\partial}$ according to different notation).

Proof: We have

$$
\begin{aligned}
d_{z}^{\prime} W=W\left[B^{-1}(z, \bar{z})(-D(d z, \bar{z})+Q(z, d z) Q(\bar{z})) B^{-1}(z, \bar{z}) v, \vec{v}\right)- \\
\left.-\operatorname{tr} B^{-1}(z, \ddot{z})(-D(d z, \bar{z})+Q(z, d z) Q(\bar{z}))\right] .
\end{aligned}
$$

Using JP31 (see [2, Appendix], or [3]) this becomes

$$
d_{z}^{\prime} W=W\left[\left(B^{-1}(z, \bar{z}) D\left(d z, \overline{z^{\bar{z}}}\right) v, \bar{v}\right)+\left(d z, \overline{z^{\bar{z}}}\right)\right] .
$$

On the other hand, we find

$$
d_{v}^{\prime} W=-W\left(B^{-1}(z, \bar{z}) d v, v\right)
$$

whence

$$
d_{z}^{\prime} W=d_{v}^{\prime} W\left(D\left(v, \overline{z^{\bar{z}}}\right) d z\right)+W\left(d z, \overline{z^{\bar{z}}}\right) .
$$

Using the lemma we can now integrate by parts in (2), exactly as in the same formula in Section 1. This yields the following result. 
Proposition. The connection in $F$ is given by

$$
\nabla f=d_{z} f-D\left(v, \overline{z^{\bar{z}}}\right)^{\top} d_{v}^{\prime} f
$$

More explicitly, the last term stands for the linear functional $d_{z}^{\prime} f$ evaluated with the argument $D\left(v, \overline{z^{\bar{z}}}\right) d z\left(=\left\{d z, \overline{z^{\bar{z}}}, v\right\}\right)$.

(Here the symbol ${ }^{\top}$ stands for the transpose of an operator. We are using subscripts in order to indicate which variable we are differentiating with respect to).

We can likewise determine the curvature.

Corollary. The corresponding curvature form is

$$
\Omega=-D\left(v, \overline{B(z, \bar{z})^{-1} d z}\right)^{\top} d_{v}^{\prime} f
$$

Proof: This follows readily from the following lemma, which may be of independent interest.

Lemma. $d^{t} z^{\bar{z}}=B(z, \bar{z})^{-1} d z$.

Proof: We have

$$
z^{\bar{z}}=B(z, \bar{z})^{-1}(z-Q(z) \bar{z})
$$

It follows that

$$
\begin{aligned}
d^{\prime} z^{\bar{z}}= & B^{-1}(z, \bar{z})(d z-Q(z, d z) \bar{z})- \\
& -B(z, \bar{z})^{-1}(-D(d z, \bar{z})+Q(z, d z) Q(\bar{z})) B(z, \bar{z})^{-1}(z-Q(z) \bar{z}) .
\end{aligned}
$$

Using once more JP31 the last term can be written

$$
B(z, \bar{z})^{-1} D\left(d z, \overline{z^{\bar{z}}}\right)(z-Q(z) \bar{z}),
$$

which again in view of JP32 ([2, Appendix $],[\mathbf{3}])$ is

$$
B(z, \bar{z})^{-1} D(d z, \bar{z}) \text {. }
$$

If we combine this with the first term, we obviously get the desired expression. 
We can now investigate horizontal sections in a similar way as in Section 2. Seeking solutions of the type

$$
f_{t}(v)=f(\phi(t) v)
$$

with $\phi(t) \in \mathrm{GL}(V)$, one is lead to the differential equation

$$
d \phi=\phi D\left(z^{\prime}(t), \overline{z(t)^{\overline{z(t)}}}\right) d t .
$$

Remark. As in the remark in Section 2, the bundle $F$ comes as sum of certain bundles $F(n)(n=0,1, \ldots)$,

$$
F=\sum_{n=0}^{\infty} \oplus^{\circ} F(n) .
$$

But now the $F(n)$ are not line bundles, but (holomorphic) vector bundles of higher rank; the sections of $F(n)$, for any given $n$, can be viewed as symmetric differential forms of degree $n$ and are represented by holomorphic functions $f(z, v)$ in the variables $z$ and $v$ which are homogeneous polynomials of degree $n$ in $v$. This allows to make an interesting (and unexpected) application. Namely, we can determine the Hermitean connection in any of the bundies $F(n)$, in particular, thus in $F(1)$, which is the same thing as the complex cotangent bundle $T^{*} \mathcal{D}$ of $\mathcal{D}$. In particular, from (5) we can draw the conclusion that the holonomy group of $\mathcal{D}$ (in the Bergman metric) is $\mathbf{H}$, the complexification of $\mathbf{K}$, the isotropy group of the origin 0 in $\mathrm{G}_{0}$ (see [2]).

5. A general framework for the bundles of Fock and Fischer. Compairing the construction in this paper with the definition of the Fock bundle(s) in [4]-[7] suggests to consider the following set-up: a complex manifold $M$, an Hermitean vector bundle $E$ over $M$ and, on top of everything; an Hermitean line bundle $L$ over $E$, as depicted by the following diagram:

$$
M \stackrel{p_{1}}{\longleftarrow} E \stackrel{p_{2}}{\longleftarrow} L .
$$

However, $L$ is not supposed to be arbitrary but of a very special kind, its definition taking into account the bundle structure of $E$. Namely, we assume that it is defined by a family of (holomorphic) transition functions $\left\{g_{\alpha \beta}\right\}$, corresponding to an open covering of $E$ by sets of the form $p_{1}^{-1}\left(V_{\alpha}\right)$, where cach $V_{\alpha}$ is an open subset of the manifold $M$, in other words a "cylinder", such that

$$
g_{\alpha \beta}(z, v)=e^{L_{\alpha \beta}(z, v)} N_{\alpha \beta}(z),
$$


where it is assumed that the function $L_{\alpha \beta}(z, v)$ is quadratic in the argument $v$. (Here, somewhat abusively, we have written $(z, v)$ for a general element of $E: z \in M, v \in E$ while, $p_{1}(v)=z$ ). In addition, we assume that the metric in $L$ is defined by a family of smooth functions $\left\{h_{\alpha}\right\}$ of the form

$$
h_{\alpha}(z, v)=e^{-F_{\alpha}(z, v)} M_{a}(z),
$$

where $F_{\alpha}(z, v)$ is quadratic in $v$ (but not necessarily holomorphic). As we must have $h_{\alpha}=\left|g_{\alpha \beta}\right|^{2} h_{\beta}$, it follows that

$$
\begin{aligned}
F_{\alpha}(z, v)+2 \operatorname{Re} L_{\alpha \beta}(z, v) & =F_{\beta}(z, v), \\
\left|M_{\alpha \beta}(z)\right|^{2} N_{\alpha}(z) & =N_{\beta}(z) .
\end{aligned}
$$

Moreover, one has $g_{\alpha \beta} \cdot g_{\beta \gamma}=g_{\alpha \gamma}$. If follows, in particular, that the functions $N_{\alpha \beta}$, in turn, may be viewed as the transition functions of a line bundle $\Lambda$, say, over $M$. A natural choice for $\Lambda$ seems to be a suitable power of the volume bundle of $M$ (a "Berezin bundle").

So far we have not investigated this general framework in any detail.

Remark. Lastly, let us mention that in the literature there is something called the "Weyl bundle" (see e. g. [8]). It is an object associated with any "symplectic bundle" (a vector bundle over a paracompact space whose fibers are symplectic vector spaces) but its definition is rather abstract involving $C^{*}$-algebra theory. It is not clear that it has any bearing to what we are speaking of here.

\section{References}

1. J. Arazy, A survey of invariant Hilbert spaces of analytic functions in a bounded symmetric domain, Technical report, no. 19. Mittag-Leffler Institute, 1990/91.

2. O. Loos, Bounded symmetric domains and Jordan pairs, Department of Math., Univ. of Calif., Irvine, 1977.

3. O. Loos, "Jordan pairs," Lecture Notes in Math. 460, SpringerVerlag, Berlin-Heidelberg-New York, 1975.

4. J. PEETRE, The Fock bundle, in "Analysis and partial differential equations," Cora Sadosky (ed.), A collection of papers dedicated to Mischa Cotlar, Marcel Dekker, New York and Basel, 1990, pp. 301-326.

5. J. Peetre, The periodic Fock bundle, Algebra i Analiz 3 (1992), $134-154$.

6. J. PeETRE, The doubly periodic Fock bundle, in preparation. 
7. J. PeEtre, Cartan domains, symplectic spaces, and Fock bundle, in preparation.

8. R. J. PLYMEN, The Weyl bundle, J. Funct. Anal. 49 (1982), 186. 197.

Matematiska institutionen

Lunds universitet

Box 118

$\mathrm{S}-22100$ Lund

SWEDEN

Primera versió rebuda el 3 d'Agost de 1992, darrera versió rebuda el 18 de Novembre de 1992 